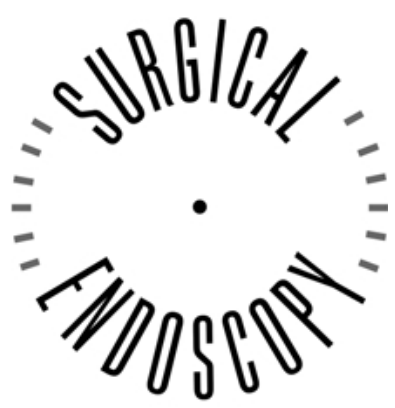

and Other Interventional Techniques

\title{
Changing pattern of the intraoperative blood pressure during endoscopic adrenalectomy in patients with Conn's syndrome
}

I. Gockel, ${ }^{1}$ A. Heintz, ${ }^{1}$ R. Kentner, ${ }^{2}$ C. Werner, ${ }^{2}$ Th. Junginger ${ }^{1}$

${ }^{1}$ Department of General and Abdominal Surgery, Johannes Gutenberg-University, Langenbeckstr. 1, Mainz, 55101, Germany

${ }^{2}$ Department of Anaesthesiology, Johannes Gutenberg-University, Langenbeckstr. 1, Mainz, 55101, Germany

[Surg Endosc (2005) 19: 1491-1497, DOI: 10.1007/s00464-004-2286-0]

C. Werner's name was misspelled in the list of authors for this article. The name is corrected here. 